\title{
Using Societal Values to Inform Public Health Policy During the COVID-19 Pandemic: The Role of Health Preference Research
}

\author{
Rachael L. DiSantostefano ${ }^{1}\left[\right.$ (1) Fern Terris-Prestholt ${ }^{2}{ }^{(1)}$
}

Accepted: 2 April 2021 / Published online: 22 April 2021

(c) The Author(s), under exclusive licence to Springer Nature Switzerland AG 2021

\section{Editorial Introducing this Special Issue}

Since March 2020, the severe acute respiratory syndrome coronavirus 2 (SARS-CoV-2; coronavirus disease 2019 [COVID-19]) pandemic has had a substantial effect on daily life worldwide. All countries have experienced both significant threats to health and social and economic disruption. COVID-19 infections and resulting morbidity and mortality have overwhelmed health systems, affected in-person learning in schools, and changed how and when individuals can shop and patronize businesses. Global responses to COVID19 have varied considerably in terms of trade-offs between control measures and keeping business as usual for as long as possible. As the pandemic continues through 2021, the availability and administration of vaccines will again influence policy and control measures.

In this special issue of The Patient, we highlight seven empirical COVID-19 health preference research studies to better understand the value of health and health-related policies around the COVID-19 pandemic. Given the rapid evolution of the disease trends and control strategies, each study should be interpreted in the context of the time of collection. Studies in this special issue examine trade-offs in the pandemic relating to early personal restrictions and lockdowns, the allocation of intensive care unit (ICU) beds and ventilators during scarcity, and the acceptance of vaccines with various attributes, including ways that uptake might be maximized. Understanding community preferences can inform government policies in these areas.

Rachael L. DiSantostefano

rdisanto@its.jnj.com

Fern Terris-Prestholt

Fern.Terris-Prestholt@1shtm.ac.uk

Janssen Research \& Development, LLC, Titusville, NJ, USA

2 United Nations Joint Programme on HIV/AIDS, Geneva, Switzerland

\section{Disease Control Strategies Early in Outbreak}

Using discrete-choice experiments, two preference studies early in the pandemic evaluated various lockdown policies to control the spread of COVID-19 in Singapore and Australia.

In April 2020, a study evaluated the acceptability of different potential government control policies depending upon the severity of the local and global spread of COVID-19 in Singapore [1]. Five policies restricted local movement (no gatherings of more than 50 people, school closure, work from home orders, shut down public transportation, lockdown) and four controlled borders (quarantine-restricted countries, quarantine-all countries, no entry-restricted countries, no entry-all countries). The study showed support for restrictions in local movement, which was heavily driven by case fatality rate. The degree of global spread was the most important factor for support of border control policies. Support for different policies varied based on patient characteristics.

In May 2020, a study evaluated the acceptability of different COVID-19 control measures in Australia [2]. Attributes included control measures (restriction level, duration of restrictions, tracking of people), burden of disease (number of people infected with disease, total number of deaths), and economic consequences (number of people who lose their job, additional government spending, additional income tax levy for the next three years). Participants demonstrated that policies resulting in a high death toll were less acceptable than those resulting in high economic losses (unemployment, government expenditure, or tax levies). However, lower unemployment and government expenditure were also important. There was heterogeneity of preferences by characteristics. Interestingly, the use of mobile phones and wearables to manage and contain disease spread at the potential expense of privacy were preferred over not using them in this Australian sample. This is consistent with another COVID-19 discrete-choice experiment in Australia 
demonstrating that acceptance of such tracking via surveillance technology may depend on the context and the state of the pandemic [3]. Another discrete-choice experiment estimated uptake of digital technology for disease tracking at about $60 \%$ [4]. However, the use of mobile phones to track individuals may be met with mixed acceptance within and across countries and raises ethical issues [5].

It is not surprising that the severity of the outbreak, in particular COVID-19 deaths, was the most important attribute in accepting restrictions across both studies examining disease control strategies. In addition, heterogeneity of preferences, based on participant characteristics, for different COVID-19 disease control measures was identified in both a survey of more than 7000 people across seven European countries and a preference study in the USA $[6,7]$. Understanding this preference heterogeneity can aid in the design of lockdown policies to achieve greater adherence, including tailoring to different populations.

\section{Allocation Decisions with Ventilator and Intensive Care Unit Bed Shortage}

Early in the pandemic, countries with the highest infection rates experienced shortages of protective equipment for healthcare workers, ventilators, and ICU beds, requiring many health systems to develop rationing policies and triage to ensure equitable distribution to those with greatest benefit and need $[8,9]$. Two discrete-choice experiments evaluated prioritization of ventilators and ICU beds across patients with varying characteristics during shortages due to spikes in COVID-19 infection.

In August 2020, an Australian study explored preferences for allocating ventilators to ICU patients during a pandemic in the event of demand for ventilators exceeding supply [10]. This study used a discrete-choice experiment to elicit general population preferences, with respondents asked to choose which of two patients in need would get the ventilator. Patient attributes included age, chance of survival if ventilated, gender, smoking status, dependents, healthcare worker employment, and disability status. On average, patients prioritized for the ventilator were younger, were more likely to survive, were non-smokers, had dependents, were healthcare workers, and were without disability. Though the background number of cases at the time of data collection was low, results could inform allocation policies in times of scarcity [11-14].

Along with shortages of ventilators, there have also been shortages of ICU beds, resulting in extensive expansions in capacity and predictive modeling of need $[15,16]$. Gijsbers et al. [17] conducted a discrete-choice experiment in October 2020 to examine the public's perspective on priority setting in ICU admissions. Attributes of patients who might require the ICU included age, profession, guardianship (e.g., dependents), ICU length of stay, risk-conscious behavior on a societal level (e.g., adherence to national safety measures such as wearing a mask or social distancing) and individual health-conscious behavior (e.g., obesity, smoking, exercise, etc.). Societal-level risk-conscious behavior was the most important nonmedical consideration in prioritizing patients, followed by patient age and individual health-conscious behavior. Younger respondents prioritized younger patients.

These studies demonstrated similarities in societal values for health resource allocation between Australia and the Netherlands, with higher value placed on individuals who were younger, had dependents, and exhibited healthy behaviors. Healthcare allocation studies generally find that the highest value is placed on saving the most lives. However, there can be potential value differences among individuals with respect to the value placed on treating the "sickest first" (worst prospects if left untreated) versus the "youngest first" (potential to live longest if cured) in allocation criteria depending on the context [18]. Studies in other healthrelated areas have also shown that societal views penalize individuals who may be seen as contributing to their poor health state based on their behavioral choices (e.g., liver transplant allocation to those with alcohol-induced cirrhosis) [19].

\section{Vaccine Hesitancy and Vaccine Uptake}

With the approval of a number of highly effective vaccines, providing a light at the end of the tunnel, perhaps the biggest public health concern relating to ending the COVID-19 crisis is achieving sufficient availability, distribution, and uptake of COVID-19 vaccines to achieve herd immunity [20-23]. Lack of trust in the efficacy and safety of vaccines may inhibit the ability to reach herd immunity, with many factors contributing to vaccine hesitancy across the globe [24-28].

In May 2020, Tervonen et al. [29] conducted a global study within the first seven weeks of the first global lockdown to examine participants' willingness to wait for a highly effective vaccine (i.e., a vaccine that will work in everyone vaccinated) versus taking a vaccine sooner that would work in only $50 \%$ of the people vaccinated. Their findings suggested that governments and regulators should consider efficacy over speed, as fast approvals of vaccines that are only $50 \%$ effective may affect not only vaccine uptake but also the public's trust in vaccine programs and policies into the future. Another preference study found that less than $50 \%$ of participants were willing to accept a vaccine with less rigorous testing [30]. As has been much discussed, vaccination programs will succeed only if there is widespread 
belief that available vaccines are safe and effective and have been rigorously tested without cutting any corners [31-37].

In November 2020, just after the US presidential election and with increasing numbers of daily cases, Craig [38] examined preferences for vaccination to inform vaccine policies in the USA relating to maximizing uptake and understanding who is likely to display vaccine hesitancy (e.g., planning to not get a vaccine). The authors' survey included closed-ended questions about plans to get a vaccine and a discrete-choice experiment among those who expressed openness to getting a vaccine. The results of their study identified groups who are less likely to get vaccinated, the potential value of a vaccination card, and different preferences regarding the vaccine setting (office vs. community). The authors found that, if the vaccination program offers a vaccination card, a choice of setting, and the most effective vaccine, uptake will be maximized among those willing to get a vaccine in the USA. Not surprisingly, a vaccine card may produce a meaningful increase in uptake given its potential value. Such a vaccine or immunity card is controversial given many unknowns about COVID-19 immunity, ethical considerations, and inequities in who might seek or have access to the vaccine $[24,39-43]$ as well as the potential for fraud [44]. Although whether such a card would be mandatory for future air travel or entry to school, etc. is uncertain, proof of COVID-19 vaccination may be essential for health and nonhealth uses as vaccines become available. Recently, the European Commission proposed a Digital Green Certificate to facilitate travel within the EU during the pandemic [45].

\section{Quality-Adjusted Life-Years of COVID-19 Health States}

Relating to policy setting, Poteet and Craig [46] used the same panel as Craig [38] in the USA to estimate qualityadjusted life-year values for COVID-19 health states, including asymptomatic and symptomatic COVID-19, observed outside of the hospital setting. To our knowledge, this is the first study to provide utility values for COVID-19 health states, which can be used for health economic assessment of interventions and policy decisions explored in this special issue, for example, lockdown policies [47].

We hope readers of this journal find this special issue valuable in understanding how the preference studies herein could inform healthcare treatment and policies during the COVID-19 pandemic. Importantly, the preferences expressed in these studies were informed by what was known at the point in time that they were conducted. There was substantial preference heterogeneity across people. This is a key area that warrants further investigation to inform targeting strategies to support positive behavior change across populations. As the scientific knowledge around COVID-19 infection has evolved, so have disease outbreaks, restrictions, and restriction fatigue. None of the preference studies in this special issue examined the use of masks, which were initially controversial but emerged as one of the most important factors to prevent community spread [48-50]. The biggest tension to emerge over the last year has been between balancing the utility of lives saved among the vulnerable and disproportionately elderly versus the social, educational, and economic opportunity costs predominately among younger individuals. It would be worthwhile to repeat or conduct additional preference studies as the COVID-19 pandemic context evolves. In the future, preference studies may inform policies on how restrictions might be lifted as society returns to a "new normal" and vaccines become more widely available.

\section{Declarations}

Funding No sources of funding were used to conduct this study or prepare this manuscript.

Conflict of interest Rachael DiSantostefano is employed by Janssen Research and Development, LLC, and is a stockholder in Johnson \& Johnson. Fern Terris-Prestholt has no conflicts of interests that are directly relevant to the content of this article.

Disclaimer This text and its contents reflect the views of the authors and not those of the authors' respective organizations.

Availability of data and material Not applicable.

Ethics approval Not applicable.

Consent Not applicable.

Author contributions RD created the first draft. All authors reviewed and provided comments on the draft and approved the final version before submission.

\section{References}

1. Ozdemir S, Tan SNG, Chaudhry I, et al. Public preferences for government response policies on outbreak control. Patient. 2021. https://doi.org/10.1007/s40271-020-00494-9.

2. Manipis K, Street D, Cronin P, et al. Exploring the trade-off between economic and health outcomes during a pandemic: a discrete choice experiment of lockdown policies in Australia. Patient. 2021. https://doi.org/10.1007/s40271-021-00503-5.

3. Degeling $\mathrm{C}$, et al. Changes in public preferences for technologically enhanced surveillance following the COVID-19 pandemic: a discrete choice experiment. BMJ Open. 2020;10(11):e041592.

4. Jonker M, et al. COVID-19 contact tracing apps: predicted uptake in the Netherlands based on a discrete choice experiment. JMIR Mhealth Uhealth. 2020;8(10):e20741.

5. Cohen IG, Gostin LO, Weitzner DJ. Digital smartphone tracking for COVID-19: public health and civil liberties in tension. JAMA. 2020;323(23):2371-2. 
6. Sabat I, et al. United but divided: policy responses and people's perceptions in the EU during the COVID-19 outbreak. Health Policy. 2020;124(9):909-18.

7. Reed S, Gonzalez JM, Johnson FR. Willingness to accept tradeoffs among COVID-19 cases, social-distancing restrictions, and economic impact: a nationwide US study. Value Health. 2020;23(11):1438-43.

8. Emanuel EJ, et al. Fair allocation of scarce medical resources in the time of Covid-19. N Engl J Med. 2020;382(21):2049-55.

9. Maves RC, et al. Triage of scarce critical care resources in COVID-19 an implementation guide for regional allocation: an expert panel report of the Task Force for Mass Critical Care and the American College of Chest Physicians. Chest. 2020;158(1):212-25.

10. Norman R, Robinson S, Dickinson H, et al. Public preferences for allocating ventilators in an intensive care unit: a discrete choice experiment. Patient. 2021. https://doi.org/10.1007/ s40271-021-00498-z.

11. Han SA, Koch VG. Clinical and ethical considerations in allocation of ventilators in an influenza pandemic or other public health disaster: a comparison of the 2007 and 2015 New York State Ventilator Allocation Guidelines. Disaster Med Public Health Prep. 2020;14(6):e35-44. https://doi.org/10.1017/dmp.2020.232.

12. Piscitello GM, et al. Variation in ventilator allocation guidelines by US state during the coronavirus disease 2019 pandemic: a systematic review. JAMA Netw Open. 2020;3(6):e2012606.

13. Reynolds JM, Guidry-Grimes L, Savin K. Against personal ventilator reallocation. Camb Q Healthc Ethics. 2021;30(2):27284. https://doi.org/10.1017/S0963180120000833.

14. Yahya AS, Khawaja S. Medical ethics and ventilator allocation during the COVID-19 pandemic. Prim Care Companion CNS Disord. 2020;22(4):20com02687. https://doi.org/10.4088/PCC. 20com02687

15. Bauer J, et al. Access to intensive care in 14 European countries: a spatial analysis of intensive care need and capacity in the light of COVID-19. Intensive Care Med. 2020;46(11):2026-34.

16. Shoukat A, et al. Projecting demand for critical care beds during COVID-19 outbreaks in Canada. CMAJ. 2020;192(19):E489-e496.

17. Gijsbers M, Keizer IE, Schouten SE, et al. Public preferences in priority setting when admitting patients to the ICU during the COVID-19 crisis: a pilot study. Patient. 2021. https://doi.org/10. 1007/s40271-021-00504-4.

18. Grover S, McClelland A, Furnham A. Preferences for scarce medical resource allocation: differences between experts and the general public and implications for the COVID-19 pandemic. $\mathrm{Br}$ J Health Psychol. 2020;25(4):889-901.

19. Ubel PA, et al. Allocation of transplantable organs: do people want to punish patients for causing their illness? Liver Transpl. 2001;7(7):600-7.

20. Burki TK. Herd immunity for COVID-19. Lancet Respir Med. 2021;9(2):135-6. https://doi.org/10.1016/S2213-2600(20) 30555-5.

21. Clemente-Suárez VJ, Hormeño-Holgado A, Jiménez M, BenitezAgudelo JC, Navarro-Jiménez E, Perez-Palencia N, Maestre-Serrano R, Laborde-Cárdenas CC, Tornero-Aguilera JF. Dynamics of population immunity due to the herd effect in the COVID-19 pandemic. Vaccines (Basel). 2020;8(2):236. https://doi.org/10. 3390/vaccines 8020236 .

22. Frederiksen LSF, et al. The long road toward COVID-19 herd immunity: vaccine platform technologies and mass immunization strategies. Front Immunol. 2020;11:1817.

23. Kwok KO, et al. Herd immunity-estimating the level required to halt the COVID-19 epidemics in affected countries. J Infect. 2020;80(6):e32-3.
24. Phelan AL. COVID-19 immunity passports and vaccination certificates: scientific, equitable, and legal challenges. Lancet. 2020;395(10237):1595-8.

25. Dror AA, et al. Vaccine hesitancy: the next challenge in the fight against COVID-19. Eur J Epidemiol. 2020;35(8):775-9.

26. Harrison EA, Wu JW. Vaccine confidence in the time of COVID19. Eur J Epidemiol. 2020;35(4):325-30.

27. McAteer J, Yildirim I, Chahroudi A. The VACCINES act: deciphering vaccine hesitancy in the time of COVID-19. Clin Infect Dis. 2020;71(15):703-5.

28. Puri N, et al. Social media and vaccine hesitancy: new updates for the era of COVID-19 and globalized infectious diseases. Hum Vaccin Immunother. 2020;16(11):2586-93.

29. Tervonen T, Jimenez-Moreno AC, Krucien N, et al. Willingness to wait for a vaccine against COVID-19: results of a preference survey. Patient. 2020. https://doi.org/10.1007/s40271-020-00483-y.

30. Goldman RD, et al. Caregivers' willingness to accept expedited vaccine research during the COVID-19 pandemic: a cross-sectional survey. Clin Ther. 2020;42(11):2124-33.

31. González-Melado FJ, Di Pietro ML. The vaccine against COVID-19 and institutional trust. Enferm Infecc Microbiol Clin. 2020. https://doi.org/10.1016/j.eimc.2020.08.001.

32. Mahase E. Covid-19: Vaccine trials need more transparency to enable scrutiny and earn public trust, say experts. BMJ. 2020;371:m4042.

33. Ojikutu BO, Stephenson KE, Mayer KH, Emmons KM. Building trust in COVID-19 vaccines and beyond through authentic community investment. Am J Public Health. 2021;111(3):366-8. https://doi.org/10.2105/AJPH.2020.306087.

34. Opel DJ, Salmon DA, Marcuse EK. Building trust to achieve confidence in COVID-19 vaccines. JAMA Netw Open. 2020;3(10):e2025672.

35. Schwartz JL. Evaluating and deploying Covid-19 vaccines - the importance of transparency, scientific integrity, and public trust. N Engl J Med. 2020;383(18):1703-5.

36. Trogen B, Oshinsky D, Caplan A. Adverse consequences of rushing a SARS-CoV-2 vaccine: implications for public trust. JAMA. 2020;323(24):2460-1.

37. Warren RC, et al. Trustworthiness before trust-covid-19 vaccine trials and the black community. N Engl J Med. 2020;383(22):e121.

38. Craig BM. United States COVID-19 vaccination preferences (CVP): 2020 hindsight. Patient. 2021.https://doi.org/10.1007/ s40271-021-00508-0

39. Brown $\mathrm{RCH}$, et al. The scientific and ethical feasibility of immunity passports. Lancet Infect Dis. 2021;21(3):e58-63. https://doi. org/10.1016/S1473-3099(20)30766-0.

40. Brown RCH, et al. Passport to freedom? Immunity passports for COVID-19. J Med Ethics. 2020;46(10):652-9.

41. Kofler N, Baylis F. Ten reasons why immunity passports are a bad idea. Nature. 2020;581(7809):379-81.

42. Persad G, Emanuel EJ. The ethics of COVID-19 immunity-based licenses ("immunity passports"). JAMA. 2020;323(22):2241-2.

43. Voo TC, Clapham H, Tam CC. Ethical implementation of immunity passports during the COVID-19 pandemic. J Infect Dis. 2020;222(5):715-8.

44. Tidy J. Covid-19: vaccines and vaccine passports being sold on darknet. 2021 https://www.bbc.co.uk/news/technology-56489574. Accessed 30 Mar 2021.

45. European Commission. Coronavirus: Commission proposes a Digital Green Certificate [media release]. 2021 Mar 17. https:// ec.europa.eu/commission/presscorner/detail/en/ip_21_1181.

46. Poteet S, Craig BM. QALYs for COVID-19: a comparison of US EQ-5D-5L value sets. Patient. 2021. https://doi.org/10.1007/ s40271-021-00509-z. 
47. Donaldson C, Mitton C. Coronavirus: where has all the health economics gone? Int J Health Policy Manag. 2020;9(11):466-8.

48. Eikenberry SE, et al. To mask or not to mask: modeling the potential for face mask use by the general public to curtail the COVID19 pandemic. Infect Dis Model. 2020;5:293-308.
49. Lyu W, Wehby GL. Community use of face masks and COVID19: evidence from a natural experiment of state mandates in the US. Health Aff (Millwood). 2020;39(8):1419-25.

50. Matuschek $\mathrm{C}$, et al. Face masks: benefits and risks during the COVID-19 crisis. Eur J Med Res. 2020;25(1):32. 